\title{
Elevated rheumatoid factor and long term risk of rheumatoid arthritis: a prospective cohort study
}

\author{
@ @® OPEN ACCESS
}

\author{
Sune F Nielsen senior scientist ${ }^{12}$, Stig E Bojesen consultant ${ }^{123}$, Peter Schnohr consultant ${ }^{3}$, Børge \\ G Nordestgaard professor ${ }^{123}$
}

${ }^{1}$ Department of Clinical Biochemistry, 54M1, Herlev Hospital, Copenhagen University Hospital, Herlev Ringvej 75, DK-2730 Herlev, Denmark; ${ }^{2}$ Faculty of Health Sciences, University of Copenhagen, Denmark; ${ }^{3}$ The Copenhagen City Heart Study, Bispebjerg Hospital, Copenhagen University Hospital

\begin{abstract}
Objective To test whether elevated concentration of rheumatoid factor is associated with long term development of rheumatoid arthritis.

Design A prospective cohort study, the Copenhagen City Heart Study. Blood was drawn in 1981-83, and participants were followed until 10 August 2010

Setting Copenhagen general population.

Participants 9712 white Danish individuals from the general population aged 20-100 years without rheumatoid arthritis at study entry.

Main outcome measures Rheumatoid arthritis according to baseline plasma IgM rheumatoid factor level categories of 25-50, 50.1-100, and $>100$, versus $<25 \mathrm{IU} / \mathrm{mL}$.
\end{abstract}

Results Rheumatoid factor levels were similar from age 20 to 100 years. During 187659 person years, 183 individuals developed rheumatoid arthritis. In healthy individuals, a doubling in levels of rheumatoid factor was associated with a 3.3-fold ( $95 \%$ confidence interval 2.7 to 4.0 ) increased risk of developing rheumatoid arthritis, with a similar trend for most other autoimmune rheumatic diseases. The cumulative incidence of rheumatoid arthritis increased with increasing rheumatoid factor category $\left(P_{\text {trend }}<0.0001\right)$. Multivariable adjusted hazard ratios for rheumatoid arthritis were 3.6 (95\% confidence interval 1.7 to 7.3$)$ for rheumatoid factor levels of $25-50 \mathrm{IU} / \mathrm{mL}, 6.0$ (3.4 to 10) for $50.1-100$ $\mathrm{IU} / \mathrm{mL}$, and 26 (15 to 46 ) for $>100 \mathrm{IU} / \mathrm{mL}$, compared with $<25 \mathrm{IU} / \mathrm{mL}$ $\left(P_{\text {trend }}<0.0001\right)$. The highest absolute 10 year risk of rheumatoid arthritis of $32 \%$ was observed in 50-69 years old women who smoked with rheumatoid factor levels $>100 \mathrm{lU} / \mathrm{mL}$.

Conclusion Individuals in the general population with elevated rheumatoid factor have up to 26 -fold greater long term risk of rheumatoid arthritis, and up to $32 \% 10$ year absolute risk of rheumatoid arthritis. These novel findings may lead to revision of guidelines for early referral to a rheumatologist and early arthritis clinics based on rheumatoid factor testing.

\section{Introduction}

Rheumatoid arthritis is an autoimmune disease affecting 0.5-2\% of the population. ${ }^{12}$ Although modern treatments for rheumatoid arthritis can induce remission in many patients, diagnosis of rheumatoid arthritis in early disease stages is important for preventing irreversible damage to the synovial lining and cartilage of diseased joints and for preventing progression into later disease stages. ${ }^{3-7}$ At present, there is no good clinical available indicator for long term development of rheumatoid arthritis. ${ }^{8-10}$

Rheumatoid factor is an autoantibody targeting the $\mathrm{Fc}$ region of IgG antibodies. ${ }^{1}$ Testing for rheumatoid factor is the most widely used blood test in the classification of rheumatoid arthritis. ${ }^{311}$ In the current classification criteria for rheumatoid arthritis "definite rheumatoid arthritis" is based on the confirmed presence of synovitis in at least one joint, absence of an alternate diagnosis better explaining the synovitis, and achievement of a total score of $\geq 6$ (of a possible 10) on a scoring system. The score is derived from four criteria: the number and site of affected joints (range 0-5), serological abnormality (elevated levels of rheumatoid factor or anti-citrullinated protein antibody; range 0-3), elevated acute phase response (range 0-1), and symptom duration ( $<6 v \geq 6$ weeks; range $0-1$ ). It is often stated that levels of rheumatoid factor increase with age, ${ }^{1}$ but convincing data for this statement is difficult to find. About $80 \%$ of all patients with rheumatoid arthritis will eventually be seropositive for rheumatoid factor, while only $40 \%$ are positive at clinical onset of the disease. ${ }^{1012}{ }^{13}$ However, it is unknown whether elevated levels of rheumatoid factor in individuals in the general population without rheumatoid arthritis is associated with later development of rheumatoid arthritis.

We tested the hypothesis that elevated levels of rheumatoid factor is associated with long term development of rheumatoid 
arthritis. For this purpose, we measured baseline plasma levels of IgM rheumatoid factor in 9712 white Danish individuals without rheumatoid arthritis from the general population, the Copenhagen City Heart Study, and followed them for up to 28 years, during which time 183 developed rheumatoid arthritis.

\section{Methods}

The studies were approved by Herlev Hospital, Copenhagen University Hospital, and the Danish ethics committees for Copenhagen and Frederiksberg. Participants provided written informed consent.

\section{Unique identification}

The national Danish Civil Registration System records all births, immigrations, emigrations, and deaths in Denmark through the civil registration number, which uniquely identifies all inhabitants of Denmark and provides information on age and sex. ${ }^{14}$ The national Danish Civil Registration System is $100 \%$ complete-that is, for the present study all people were accounted for during the entire follow-up period.

\section{Participants}

The Copenhagen City Heart Study is a prospective study of a random sample of the Danish general population drawn using the Danish Civil Registration System and initiated in 1976-78. ${ }^{15}$ We studied white participants of Danish descent attending the 1981-83 examination: 19698 individuals aged 20-100 years were invited, 12698 (64\%) attended, and 9712 (49\%) had plasma available for measurement of rheumatoid factor in 2009-10. We excluded 52 individuals with rheumatoid arthritis diagnosed before plasma collection.

Participants filled in a questionnaire that was reviewed together with an investigator on the day of attendance. Subsequently, a physical examination was conducted and blood samples were drawn.

\section{Rheumatoid arthritis}

Participants diagnosed clinically with incident rheumatoid arthritis ${ }^{11}$ from 1 January 1977 to 10 August 2010 were identified by means of the national Danish Patient Registry, ${ }^{17}$ requiring only a single inpatient or outpatient hospital diagnosis. However, in an attempt to exclude patients with a misdiagnosis of rheumatoid arthritis, we also examined patients with at least two hospitalisations for rheumatoid arthritis at least six weeks apart in accordance with current classification criteria. ${ }^{3}$ Rheumatoid arthritis was identified using ICD-8 ((international classification of diseases, eighth revision) diagnostic codes 712.1, 712.2, 712.3 (for 1977-94) and ICD-10 codes M05-M06 (for 1995-2010). Rheumatoid arthritis end points were actively reported by hospitals nationwide immediately after discharge of patients from hospitals in the entire period of follow-up.

\section{Other autoimmune rheumatic diseases}

Participants diagnosed clinically with other incident autoimmune rheumatic diseases were identified similarly using the national Danish Patient Registry and ICD-8 and ICD-10 codes for 1977-94 and 1995-2010 respectively: systemic lupus erythematosus was coded 734.1 and L93, M32; Sjögren's syndrome was 734.90 and M32.0; systemic sclerosis was 731.0 and L94; and polymyositis or dermatomyositis was 716.0 and M33.0-M33.1.

\section{Rheumatoid factor}

Turbidity were used to measure concentrations of rheumatoid factor of IgM type in plasma (measuring range 15-440 IU/mL) (Konelab, Thermo Fischer Scientific, Helsinki, Finland). Plasma samples were drawn in $1981-83$ and frozen at $-20^{\circ} \mathrm{C}$ until measurement in 2009-10. Investigators were blinded to rheumatoid arthritis diagnoses when measuring rheumatoid factor, and blinded to rheumatoid factor level when diagnosing rheumatoid arthritis.

\section{Other covariates}

Tobacco smoking (cigarettes, pipe, cigarillos, cigars) was the daily amount of cigarettes or equivalent smoked (1 cigarette or equivalent $=20 \mathrm{~g}$ tobacco) at the examination, together with the cumulative amount of cigarettes or equivalent smoked up until examination in pack years ( 1 pack year is 20 cigarettes or equivalent smoked daily for 1 year). Body mass index (weight $(\mathrm{kg}) /\left(\right.$ height $\left.(\mathrm{m})^{2}\right)$ was measured. Alcohol use was based on self reported number of drinks per week of beer, wine, and spirits ( 1 drink $\approx 12 \mathrm{~g}$ alcohol). We categorised parity for women $(0$, $1-2$, or $>2$ children), marital status (single, married, separated or divorced, or widowed), and level of education (elementary (1-9 years' schooling), high school (10-12 years), or academic (>12 years)).

All values are as reported by individuals at study entry in 1981-83, but these covariates were also re-examined in the 1991-94 and 2001-03 examinations and used in the multivariable adjustment as time varying covariates. Information on age and sex was $100 \%$ complete, while information on other covariates was $99 \%$ complete ( $1 \%$ of participants each missed one or more covariates; see table $\Downarrow$ ). Missing information was multivariable imputed before categorisation, thus all statistical analyses were complete for all 9712 participants.

\section{Statistical analyses}

Statistical analyses were performed with Stata 12.1 SE software. All tests were two sided. We used log transformation of rheumatoid factor levels to calculate geometric means for each age group. Plasma levels of rheumatoid factor were illustrated using box plots with $1 \%, 25 \%, 50 \%, 75 \%$, and $99 \%$ on the $\log$ scale.

Receiver operator characteristics curves of plasma levels of rheumatoid factor at baseline and all future events of rheumatoid arthritis were used to determine an optimal threshold concentration of rheumatoid factor of $25 \mathrm{IU} / \mathrm{mL}$ - that is, the level above and below which individuals are best separated into those with and without future rheumatoid arthritis (see supplementary fig 1 in online data supplement). From this threshold we chose categories of rheumatoid factor of $<25$, 25-50, 50.1-100, and >100 IU/mL—cut-off values were doublings starting from $25 \mathrm{IU} / \mathrm{mL}$. For trend tests across these categories, we used the logarithm of individuals' rheumatoid factor values on a continuous scale.

First, the association between rheumatoid factor and risk of rheumatoid arthritis and other autoimmune rheumatic diseases were investigated on a continuous scale of doublings of rheumatoid factor as the predictor variable. Second, the association between rheumatoid factor and risk of rheumatoid arthritis was studied by comparing participants by categories of rheumatoid factor.

Cumulative incidence curves were estimated by the method of Kaplan-Meier and Fine-Gray, and log rank trend tests examined differences between rheumatoid factor categories. We calculated 
hazard and subhazard ratios with $95 \%$ confidence intervals by means of Cox and Fine-Gray regression models with age as the time scale, left truncation, and delayed entry at age of entering the Copenhagen City Heart Study. The Cox and Fine-Gray models were adjusted for known risk factors and markers of lifestyle and social status - that is, for age (automatic adjustment as age is time scale), sex, alcohol intake, body mass index, current daily tobacco use, cumulative tobacco use, marital status, parity, and years of education. the covariates were at study entry, but were also used as time-varying covariates at follow-up examinations in 1991-94 and 2001-03. Missing values in covariates were imputed using multivariable normal regression imputation (mi impute mvn), where age at examination, sex and birth year were independent variables, and alcohol use, body mass index, current daily smoking, cumulative tobacco use, marital status, parity and years of education were dependent variables in the model. In sensitivity analyses we examined those individuals with complete information on all covariates for the 1981-1983 examination. Supplementary table 1 in the online data supplement compares individuals with complete information against the entire cohort with respect to variables used for multivariable adjusted analysis, and lists the fraction with missing information for each covariate.

For Cox proportional hazards regression analyses, we assessed the assumption of proportional hazards graphically by plotting $\log$ (cumulative hazard) for different rheumatoid factor categories as a function of $\log (\mathrm{age})$. We detected no major violations of the proportional hazards assumption. Interaction was tested for by introducing a two factor interaction term in the Cox models.

All individuals were followed from study entry and censored at the date of emigration ( $n=52), 10$ August 2010, or diagnosis of rheumatoid arthritis $(n=183)$, whichever came first. In the Cox regression models death $(\mathrm{n}=6380)$ also led to censoring, whereas in the Fine-Gray regression models death acted as a competing event. Given that $66 \%$ of participants died during follow-up, competing risks from death could potentially bias the findings. Cox regression appropriately stops allowing individuals from contributing person-time when they died and then no longer are at risk, but this means that failure from other causes is unobservable. Fine-Gray regression is a proportional hazards model for the cumulative incidence function, where failure from other causes is observable. ${ }^{18}$ If findings from both regressions are similar, then the influence of competing events on the association between rheumatoid factor and rheumatoid arthritis is limited, which is the case in the present study. Thus, competing risk of death (information on which is $100 \%$ complete in the Danish registries) was accounted for in the analysis. The five main causes of death for the cohort (as assessed from the national Danish Causes of Death Registry) are shown in supplementary table 2 in the online data supplement, distributed according to categories of rheumatoid factor. For evaluation of risk of rheumatoid arthritis developed within 10 years of blood sampling, all surviving participants were censored after 10 years for follow-up; this was done as plasma biomarkers may have larger risk estimates for shorter follow-up than longer follow-up time.

Poisson regression models were used for estimation of absolute 10 year risks of rheumatoid arthritis. These risks were estimated for each rheumatoid factor category as the percentage within 10 years from baseline who developed rheumatoid arthritis, stratified for age, sex, and smoking. Such 10 year absolute risks are used in prediction tools for other major diseases, such as cardiovascular disease,${ }^{19}$ breast cancer, ${ }^{20}$ and prostate cancer. ${ }^{21}$

\section{Results}

We included 9712 individuals from the general population aged 20-100 years with a measured rheumatoid factor and without a prior diagnosis of rheumatoid arthritis at study entry in a 28 year follow-up from 1981-83 to 10 August 2010. During these 187654 person years of follow-up, 183 participants developed rheumatoid arthritis. Baseline characteristics are shown in table $1 \Downarrow$. Median age at diagnosis of rheumatoid arthritis was 70 years (interquartile range 63-76). The median time from providing the blood sample to developing rheumatoid arthritis was 15 years for those with rheumatoid factor levels of $<25 \mathrm{IU} / \mathrm{mL}, 12$ years for those with $25-50 \mathrm{UI} / \mathrm{mL}$, seven years for 50.1-100 $\mathrm{IU} / \mathrm{mL}$, and seven years for $>100 \mathrm{IU} / \mathrm{mL}$.

\section{Plasma levels of rheumatoid factor by age}

The median plasma level of rheumatoid factor was $18 \mathrm{IU} / \mathrm{mL}$ (supplementary fig 2 in online data supplement). Distributions of rheumatoid factor in individuals in the general population stratified in 10 year age groups showed similar levels of rheumatoid factor across all age categories (fig $1 \Downarrow$ ). Plasma levels were measured up to 28 years after blood sampling and were not reported to participants or their doctors, and consequently could not have influenced the risk estimates described below.

\section{Risk of autoimmune rheumatic diseases}

Risk of rheumatoid arthritis during the 28 years' follow-up increased by a factor of 3.3 ( $95 \%$ confidence interval $2.7 \%$ to $4.0 \%$ ) for a doubling of rheumatoid factor level (fig $2 \Downarrow$ ). The risk of the other autoimmune rheumatic diseases Sjögren's syndrome, systemic lupus erythematosus, and systemic sclerosis showed similar trends for a doubling of rheumatoid factor level, but the number of events for individual diseases were insufficient to reach statistical significance. None of the participants developed polymyositis or dermatomyositis. Sensitivity analyses restricted to participants with complete information on all covariates at baseline showed similar results (supplementary fig 3 in online data supplement).

\section{Risk of rheumatoid arthritis}

The cumulative incidence of rheumatoid arthritis as a function of age for the four categories of baseline level of rheumatoid factor $(<25,25-50,50.1-100$, and $>100 \mathrm{IU} / \mathrm{mL})$ is shown in fig $3 \Downarrow$ as Kaplan-Meier estimates (and in supplementary fig 4 in the data supplement as Fine-Gray estimates). The cumulative incidence of rheumatoid arthritis increased with increasing rheumatoid factor (log rank trend $\mathrm{P}<0.0001$ ).

During the full 28 years of follow-up, the multivariable adjusted hazard ratios for rheumatoid arthritis were 3.6 (95\% confidence interval 1.7 to 7.3 ) for rheumatoid factor levels of $25-50 \mathrm{IU} / \mathrm{mL}$, 6.0 (3.4 to 10) for 50.1-100 IU/mL, and 26 (15 to 46) for $>100$ $\mathrm{IU} / \mathrm{mL}$ compared with $<25 \mathrm{IU} / \mathrm{mL}\left(\mathrm{P}_{\text {trend }}<0.0001\right.$ ) (fig $4 \Downarrow$, top left panel). During the first 10 years of follow-up, the corresponding hazard ratios were 6.0 (2.1 to 17$)$ for $25-50$ $\mathrm{IU} / \mathrm{mL}, 14$ (6.7 to 28$)$ for $50.1-100 \mathrm{IU} / \mathrm{mL}$, and 39 (18 to 85$)$ for $>100 \mathrm{IU} / \mathrm{mL}\left(\mathrm{P}_{\text {trend }}<0.0001\right)$ (fig $4 \Downarrow$, bottom left panel). Corresponding risk estimates that took account of the competing risk of death were similar (supplementary fig 5, left hand panels, in the data supplement). There was no interaction between sex and rheumatoid factor categories on risk of rheumatoid arthritis.

In an attempt to exclude patients misclassified with rheumatoid arthritis, we also examined patients with at least two hospitalisations for rheumatoid arthritis at least six weeks apart. 
This reduced the number of patients with rheumatoid arthritis but strengthened the association between elevated rheumatoid factor and risk of rheumatoid arthritis (fig $4 \Downarrow$, right hand panels). These results were also not influenced by competing risk of death (supplementary fig 5, right hand panels). Sensitivity analyses restricted to participants with complete information on covariates at baseline also showed similar results (supplementary fig 6).

\section{Absolute 10 year risk of rheumatoid arthritis}

The highest absolute 10 year risk of rheumatoid arthritis of $32 \%$ was observed for 50-69 year old women who smoked and had rheumatoid factor levels $>100 \mathrm{IU} / \mathrm{mL}$ (fig $5 \Downarrow$, far right middle panel). This means that one out of three of such women will develop rheumatoid arthritis within 10 years from blood sampling. The lowest absolute 10 year risk of rheumatoid arthritis of $0.1 \%$ was observed for men $\geq 70$ years old with rheumatoid factor levels $<25 \mathrm{IU} / \mathrm{mL}$ irrespective of smoking status (fig $5 \Downarrow$, bottom panels far left and inner left).

\section{Discussion}

The principal findings in this study of 9712 individuals without rheumatoid arthritis recruited from the general population of Copenhagen are those with elevated levels of rheumatoid factor had up to 26-fold higher long term risk of developing rheumatoid arthritis and up to $32 \% 10$ year absolute risk of developing rheumatoid arthritis. These findings are novel. Importantly, these data do not serve as evidence that rheumatoid factor plays a causal role in the pathogenesis of rheumatoid arthritis.

\section{Mechanism and comparison with other studies}

Development of rheumatoid arthritis is thought to be an inflammatory process from early arthritis through rheumatoid arthritis and possibly to severe extra-articular rheumatoid arthritis. ${ }^{10132223}$ Among patients with early arthritis, only 40\% are seropositive for rheumatoid factor, whereas in the final stage of rheumatoid arthritis $80 \%$ of patients are seropositive. ${ }^{10}{ }^{23} \mathrm{~A}$ current debate is whether elevated levels of rheumatoid factor, elevated levels of anti-citrullinated protein antibody, variations in the PTPN22 gene, or some combination of these offer the best means of predicting short term (2-3 years) risk of rheumatoid arthritis. ${ }^{8924-31}$ However, the debate does not include long term risk prediction, simply because good evidence is lacking. Our finding that elevated rheumatoid factor levels are associated with an increased long term (up to 28 years) risk of rheumatoid arthritis provides such data. Unfortunately we do not have information available for elevated anti-citrullinated protein antibody or genetic variations in PTPN22. Our data show that elevated levels of rheumatoid factor can be present many years before the clinical manifestation of arthritis, supported by a few earlier studies. ${ }^{32-34}$

Rheumatoid factor levels are believed to increase with age in the general population, ${ }^{1}$ but we could not confirm this. Although the age of individuals with rheumatoid factor $>100 \mathrm{IU} / \mathrm{mL}$ was 62 years compared with 58 years for the reference group with rheumatoid factor $<25 \mathrm{IU} / \mathrm{mL}$, the corresponding median times to diagnosis of rheumatoid arthritis were seven years and 15 years, indicating that the higher rheumatoid factor level $>100$ $\mathrm{IU} / \mathrm{mL}$ group can be explained by a short time to diagnosis rather than by greater age. On direct investigation, we found that rheumatoid factor levels were constant across age groups from 20 years to 100 years old.
Tobacco smoking has long been known to play a role in the pathogenesis of rheumatoid arthritis. ${ }^{35}$ Smoking related alterations to the cytokine balance, stress to the immune system, and modifications of autoantibodies are strongly associated with rheumatoid arthritis. ${ }^{36}{ }^{37}$ However, we adjusted extensively for smoking_-including daily tobacco use at examination and cumulative tobacco use in pack years at baseline and as time varying covariates at follow-up examinations-so smoking is unlikely as a confounder for our observation of increased long term risk of rheumatoid arthritis in those with elevated rheumatoid factor levels.

\section{Strengths and limitations of study}

Our study has several strengths. The large study population was homogeneous and representative of the general population, the study population was well characterized, follow-up was more than 28 years, and we had $100 \%$ follow-up. Therefore, with a median age of entry to the study above 50 years, the 28 years of follow-up is well beyond the age of 70 years, when the incidence of rheumatoid arthritis peaks. ${ }^{1}$ Also, because rheumatoid factor concentrations were measured up to 28 years after blood sampling and thus not reported to participants or their doctors, these measurements did not influence ascertainment of rheumatoid arthritis during follow-up. In other words, we here study the natural course from an elevated rheumatoid factor to a clinical diagnosis of rheumatoid arthritis.

Potential limitations include selection bias; however, as we enrolled a sample from the general population randomly selected without regard to disease status, selection bias is unlikely as an explanation for our findings. Another potential limitation is the validity of the diagnostic information; however, most of the rheumatoid arthritis diagnoses in the Danish registries were confirmed,$^{38}$ and random misclassification would tend to bias the results towards the null hypothesis and therefore cannot explain the present results (as illustrated by our sensitivity analysis). Also, as rheumatoid factor enters into the classification of rheumatoid arthritis, individuals with lifelong elevated rheumatoid factor may have a heightened probability of being diagnosed with rheumatoid arthritis. Hospitalisation with a discharge diagnosis of rheumatoid arthritis can happen many years after the first diagnosis of rheumatoid arthritis while a patient is under the care of a rheumatologist or being cared for in some other outpatient setting; thus, those cases identified from a hospital discharge diagnosis may have been classified as not having rheumatoid arthritis when they were simply not hospitalised with it. Similarly, those with rheumatoid arthritis who eventually were hospitalised might have contributed substantially more person years than they should. Likewise important, the patients in this study were presumably at a later or more severe disease stage than patients consulting private rheumatologists; however, this implies that time to diagnosis in the outpatient setting would be shorter and risk estimates correspondingly higher than observed in this study. Given that the categorisation of rheumatoid factor was devised to maximise the relation between rheumatoid factor and rheumatoid arthritis, the risk estimates based on these categories could be exaggerated and need to be replicated in another study. Finally, as we studied only white people, our results may not apply to other races.

\section{Implications for clinicians and future work}

Our finding of a particularly high absolute risk of developing rheumatoid arthritis in women with elevated rheumatoid factor who smoked is yet another reason to promote cessation of smoking among such women. In addition, our finding of high risks of developing rheumatoid arthritis based on elevated levels 
of rheumatoid factor alone suggests the need for early referral to a rheumatologist or to early arthritis clinics for examination on the basis of a positive rheumatoid factor test - even in the absence of the typical arthritic joint symptoms—-because of the better response to therapy the earlier it is initiated in rheumatoid arthritis.

The superior specificity of anti-citrullinated protein antibody tests has somewhat diminished interest in rheumatoid factor at time of diagnosis of rheumatoid arthritis, but not necessarily when it comes to prediction of long term risk. Future work should include a comparison of rheumatoid factor and anti-citrullinated protein antibody tests for the long term prediction of development of rheumatoid arthritis. Also, larger studies might be able to confirm or refute that elevated rheumatoid factor is associated with future risk for other autoimmune rheumatic diseases.

Finally, our absolute risk estimates could be used for designing a randomised controlled trial studying early intervention in high risk people, by designing the inclusion criteria around patient groups with, say, a $\geq 20 \%$ elevated 10 year absolute risk of developing rheumatoid arthritis.

\section{Conclusion}

Individuals in the general population without rheumatoid arthritis but with an elevated plasma level of rheumatoid factor have up to 26-fold greater long term risk of developing rheumatoid arthritis, and up to $32 \% 10$ year absolute risk of rheumatoid arthritis.

We thank staff and participants of the Copenhagen City Heart Study for their contribution.

Contributors: SFN and BGN had full access to all the data in the study and take responsibility for the integrity of the data and the accuracy of the data analysis. SFN, SEB, and BGN designed the study. PS, SEB, and BGN collected data. SFN performed all statistical analyses. SEB and $B G N$ oversaw statistical analyses and contributed to interpretation of data. SFN wrote the first draft of the paper. SEB, PS, and BGN edited the paper critically, and all authors approved this paper in its final form. SFN and BGN are the guarantors.

Funding: This study was funded by Herlev Hospital, Copenhagen University Hospital and the Danish Heart Foundation. The sponsors had no role in the design of the study; collection, analysis, and interpretation of data; writing of the report; or decision to submit the article for publication. The views expressed in this paper are those of the authors and not those of any funding body or others whose support is acknowledged.

Competing interests: All authors have completed the ICMJE uniform disclosure form at www.icmje.org/coi_disclosure.pdf (available on request from the corresponding author) and declare: no support from any organisation for the submitted work; no financial relationships with any organisations that might have an interest in the submitted work in the previous three years; no other relationships or activities that could appear to have influenced the submitted work.

Ethical approval: The studies were approved by Herlev Hospital, Copenhagen University Hospital, and a Danish ethical committee (the Copenhagen and Frederiksberg committee Nos. KF-100.2039/9 and KF-01-144/01), and were conducted according to the Declaration of Helsinki. Participants provided written informed consent.

Data sharing: No additional data available.

Gaston JSH. Rheumatic diseases, immunological mechanisms and prospects for new therapies . University of Cambridge, 1999.

2 Neovius M, Simard JF, Askling J. Nationwide prevalence of rheumatoid arthritis and penetration of disease-modifying drugs in Sweden. Ann Rheum Dis 2011;70:624-9.
3 Aletaha D, Neogi T, Silman AJ, Funovits J, Felson DT, Bingham CO, et al. 2010 rheumatoid arthritis classification criteria: an American College of Rheumatology/European League Against Rheumatism Collaborative initiative. Arthritis Rheum 2010;62:2569-81.

4 Cohen SB. Targeting the B cell in rheumatoid arthritis. Best Pract Res Clin Rheumatol 2010;24:553-63.

5 Don BR, Kim K, Li J, Dwyer T, Alexander F, Kaysen GA. The effect of etanercept on suppression of the systemic inflammatory response in chronic hemodialysis patients. Clin Nephrol 2010;73:431-8

6 Julia A, Erra A, Palacio C, Tomas C, Sans X, Barcelo P, et al. An eight-gene blood expression profile predicts the response to infliximab in rheumatoid arthritis. PLOS One 2009;4:e7556

7 Verstappen SMM, Albada-Kuipers GAV, Bijlsma JWJ, Blaauw AAM, Schenk Y, Haanen $\mathrm{HCM}$, et al. A good response to early DMARD treatment of patients with rheumatoid arthritis in the first year predicts remission during follow up. Ann Rheum Dis 2005:64:38-43.

8 Balsa A, del Amo J, Blanco F, Caliz R, Silva L, Sanmarti R, et al. Prediction of functional impairment and remission in rheumatoid arthritis patients by biochemical variables and genetic polymorphisms. Rheumatology 2010;49:458-66.

9 Deane KD, O'Donnell CI, Hueber W, Majka DS, Lazar AA, Derber LA, et al. The number of elevated cytokines and chemokines in preclinical seropositive rheumatoid arthritis predicts time to diagnosis in an age-dependent manner. Arthritis Rheum 2010;62:3161-72.

10 Kuriya B, Cheng CK, Chen HM, Bykerk VP. Validation of a prediction rule for development of rheumatoid arthritis in patients with early undifferentiated arthritis. Ann Rheum Dis 2009;68:1482-5

11 Arnett FC, Edworthy SM, Bloch DA, Mcshane DJ, Fries JF, Cooper NS, et al. The American-Rheumatism-Association 1987 revised criteria for the classification of rheumatoid arthritis. Arthritis Rheum 1988:31:315-24.

12 Boutry N, do Carmo CCM, Flipo RM, Cotten A. Early rheumatoid arthritis and its differentiation from other joint abnormalities. Eur J Radiol 2009;71:217-24.

13 Machold KP, Stamm TA, Eberl GJM, Nell VKP, Dunky A, Uffmann M, et al. Very recent onset arthritis-clinical, laboratory, and radiological findings during the first year of disease. $J$ Rheumatol 2002;29:2278-87.

14 Pedersen CB, Gotzsche H, Moller JO, Mortensen PB. The Danish Civil Registration System. A cohort of eight million persons. Dan Med Bull 2006:53:441-9.

15 Nordestgaard BG, Benn M, Schnohr P, Tybjaerg-Hansen A. Non-fasting triglycerides and risk of myocardial infarction, ischemic heart disease, and death in men and women. JAMA 2007;298:299-308

16 Schnohr P, Jensen JS, Scharling H, Nordestgaard BG. Coronary heart disease risk factors ranked by importance for the individual and community-21 year follow-up of 12000 men and women from the Copenhagen City Heart Study. Eur Heart J 2002:23:620-6.

17 Andersen TF, Madsen M, Jorgensen J, Mellemkjoer L, Olsen JH. The Danish National Hospital Register. A valuable source of data for modern health sciences. Dan Med Bull 1999;46:263-8.

18 Fine JP, Gray RJ. A proportional hazards model for the subdistribution of a competing risk. J Am Stat Assoc 1999;94:496-509.

19 Catapano AL, Reiner Z, De Backer G, Graham I, Taskinen MR, Wiklund O, et al. ESC/EAS guidelines for the management of dyslipidaemias: the task force for the management of dyslipidaemias of the European Society of Cardiology (ESC) and the European Atherosclerosis Society (EAS). Atherosclerosis 2011;217:3-46.

20 Weischer M, Bojesen SE, Tybjaerg-Hansen A, Axelsson CK, Nordestgaard BG. Increased risk of breast cancer associated with CHEK2*1100delC. JCO 2007;25:57-63.

21 Orsted D, Nordestgaard BG, Jensen GB, Schnohr P, Bojesen SE. Prostate-specific antigen and long-term prediction of prostate cancer incidence and mortality in the general population. Eur J Cancer 2011;47:504.

22 Turesson C, Jacobsson LTH, Sturfelt G, Matteson EL, Mathsson L, Ronnelid J. Rheumatoid factor and antibodies to cyclic citrullinated peptides are associated with severe extra-articular manifestations in rheumatoid arthritis. Ann Rheum Dis 2007:66:59-64.

23 Verschueren P, Esselens G, Westhovens R. Predictors of remission, normalized physical function, and changes in the working situation during follow-up of patients with early rheumatoid arthritis: an observational study. Scand J Rheumatol 2009;38:166-72.

24 Innala L, Kokkonen H, Eriksson C, Jidell E, Berglin E, Rantapaa-Dahlqvist S. Antibodies against mutated citrullinated vimentin are a better predictor of disease activity at 24 months in early rheumatoid arthritis than antibodies against cyclic citrullinated peptides. $J$ Rheumatol 2008:35:1002-8.

25 Johansson M, Arlestig L, Hallmans G, Rantapaa-Dahlqvist S. PTPN22 polymorphism and anti-cyclic citrullinated peptide antibodies in combination strongly predicts future onset of rheumatoid arthritis and has a specificity of $100 \%$ for the disease. Scand J Rheumatol 2006;35:29.

26 Kastbom A, Strandberg G, Lindroos A, Skogh T. Anti-CCP antibody test predicts the disease course during 3 years in early rheumatoid arthritis (the Swedish TIRA project). Ann Rheum Dis 2004:63:1085-9.

27 Nishimura K, Sugiyama D, Kogata Y, Tsuji G, Nakazawa T, Kawano S, et al. Meta-analysis: diagnostic accuracy of anti-cyclic citrullinated peptide antibody and rheumatoid factor for rheumatoid arthritis. Ann Intern Med 2007;146:797-808.

28 Tamai M, Kawakami A, Iwamoto N, Arima K, Aoyagi K, Eguchi K. Contribution of anti-CCP antibodies, proximal interphalangeal joint involvement, HLA-DRB1 shared epitope, and PADI4 as risk factors for the development of rheumatoid arthritis in palindromic rheumatism. Scand J Rheumatol 2010;39:287-91.

29 Song YW, Kang EH. Autoantibodies in rheumatoid arthritis: rheumatoid factors and anticitrullinated protein antibodies. QJM 2010;103:139-46.

30 Ursum J, Bos WH, van Dillen N, Dijkmans BAC, van Schaardenburg D. Levels of anti-citrullinated protein antibodies and IgM rheumatoid factor are not associated with outcome in early arthritis patients: a cohort study. Arthritis Res Ther 2010;12(1):R8.

31 Bas S, Genevay S, Meyer O, Gabay C. Anti-cyclic citrullinated peptide antibodies, IgM and IgA rheumatoid factors in the diagnosis and prognosis of rheumatoid arthritis. Rheumatology 2003;42:677-80.

32 Rantapaa-Dahlqvist S, de Jong BAW, Berglin E, Hallmans G, Wadell G, Stenlund H, et al. Antibodies against cyclic citrullinated peptide and IgA rheumatoid factor predict the development of rheumatoid arthritis. Arthritis Rheum 2003:48:2741-9.

33 Aho K, Heliovaara M, Maatela J, Tuomi T, Palosuo T. Rheumatoid factors antedating clinical rheumatoid-arthritis. J Rheumatol 1991;18:1282-4.

34 Nielen MMJ, van Schaardenburg D, Reesink HW, Van de Stadt RJ, horst-Bruinsma IE, de Koning MHMT, et al. Specific autoantibodies precede the symptoms of rheumatoid arthritis-a study of serial measurements in blood donors. Arthritis Rheum 2004;50:380-6.

35 Fischer KM. Hypothesis: tobacco use is a risk factor in rheumatoid arthritis. Med Hypotheses 1991;34:116-7. 


\section{What is already known on this topic}

Rheumatoid arthritis is an autoimmune disease affecting $0.5-2 \%$ of the population

At present, there is no good clinical available indicator for long term risk of developing rheumatoid arthritis

\section{What this study adds}

Individuals in the general population without rheumatoid arthritis, but with an elevated plasma level of rheumatoid factor have up to 26 -fold greater long term risk of developing rheumatoid arthritis, and up to $32 \% 10$ year absolute risk of rheumatoid arthritis

These findings may lead to revision of guidelines for early referral to a rheumatologist and early arthritis clinics based on rheumatoid factor testing

36 Hutchinson D, Shepstone L, Moots R, Lear JT, Lynch. Heavy cigarette smoking is strongly associated with rheumatoid arthritis (RA), particularly in patients without a family history of RA. Ann Rheum Dis 2001;60:223-7.

37 Baka Z, Burzas E, Nagy G. Rheumatoid arthritis and smoking: putting the pieces together Arthritis Res Ther 2009;11:238.

38 Pedersen M, Klarlund M, Jacobsen S, Svendsen AJ, Frisch M. Validity of rheumatoid arthritis diagnoses in the Danish National Patient Registry. Eur J Epidemiol 2004;19:1097-103.

Accepted: 12 July 2012

\section{Cite this as: BMJ 2012;345:e5244}

This is an open-access article distributed under the terms of the Creative Commons Attribution Non-commercial License, which permits use, distribution, and reproduction in any medium, provided the original work is properly cited, the use is non commercial and is otherwise in compliance with the license. See: http://creativecommons.org/licenses/bync/2.0/ and http://creativecommons.org/licenses/by-nc/2.0/legalcode. 


\section{Table}

Table 1| Baseline characteristics of participants at entry to the Copenhagen City Heart Study (1981-83). Values are numbers (percentages) unless stated otherwise

\begin{tabular}{|c|c|c|c|c|}
\hline & \multicolumn{4}{|c|}{ Rheumatoid factor (IU/mL) } \\
\hline & $\begin{array}{c}<25 \\
(n=9294)\end{array}$ & $\begin{array}{c}25-50 \\
(n=176)\end{array}$ & $\begin{array}{c}50.1-100 \\
(n=187)\end{array}$ & $\begin{array}{c}>100 \\
(n=55)\end{array}$ \\
\hline Median (IQR) age (years) & $58(48-65)$ & $58(49-65)$ & $62(54-67)$ & $62(55-68)$ \\
\hline \multicolumn{5}{|l|}{ Sex: } \\
\hline Female & $4826(52)$ & $88(50)$ & $91(49)$ & $31(56)$ \\
\hline Male & $4468(48)$ & $88(50)$ & $96(51)$ & $24(44)$ \\
\hline Median (IQR) body mass index & $25(22-28)$ & $24(24-28)$ & $26(23-29)$ & $25(22-17)$ \\
\hline Median (IQR) No of cigarettes smoked daily (or equivalent) ${ }^{*}$ & $7(0-15)$ & $10(0-20)$ & $8(0-15)$ & $10(0-15)$ \\
\hline Median (IQR) No of pack-years cumulative smoking & $20(5-35)$ & $22(9-36)$ & $25(10-40)$ & $20(8-34)$ \\
\hline Median (IQR) No of alcoholic drinks taken weekly $\dagger$ & $4(0-12)$ & $5(0-13)$ & $4(0-14)$ & $4(0-12)$ \\
\hline \multicolumn{5}{|l|}{ Parity } \\
\hline 0 & $1177(24)$ & $20(23)$ & $20(21)$ & $6(17)$ \\
\hline $1-2$ & $2539(52)$ & $52(59)$ & $54(60)$ & $18(60)$ \\
\hline$>2$ & $1110(23)$ & $16(18)$ & $17(19)$ & $7(23)$ \\
\hline \multicolumn{5}{|l|}{ Marital status: } \\
\hline Single & $1135(12)$ & $20(12)$ & $25(14)$ & $4(7)$ \\
\hline Married & $6041(65)$ & $101(57)$ & $122(65)$ & $33(60)$ \\
\hline Seperated or divorced & $1160(13)$ & $26(15)$ & $20(10)$ & $11(20)$ \\
\hline Widowed & $958(10)$ & $29(16)$ & $20(10)$ & $7(13)$ \\
\hline \multicolumn{5}{|l|}{ Education (No of years): } \\
\hline Elementary (1-9) & $6302(68)$ & $135(77)$ & $133(71)$ & $44(80)$ \\
\hline High school (10-12) & $2522(27)$ & $36(20)$ & $47(25)$ & $10(18)$ \\
\hline Academic $(>12)$ & $440(5)$ & $5(3)$ & $7(4)$ & $1(2)$ \\
\hline
\end{tabular}

IQR=interquartile range.

Missing information were multivariable imputed ( $1 \%$ of participants each missed one or more covariates).

${ }^{*} 1$ cigarette $=20 \mathrm{~g}$ of tobacco.

t1 drink approximates to $12 \mathrm{~g}$ of alcohol.

‡Parity (No of children) is for women only. 


\section{Figures}

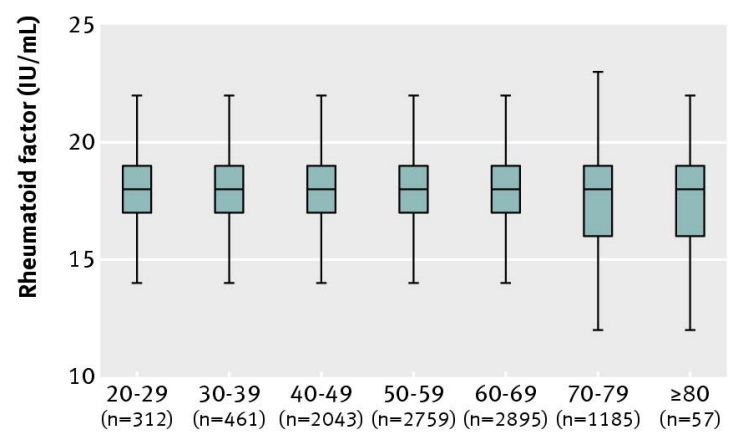

Age (years)

Fig 1 Box and whisker plots of distribution of plasma rheumatoid factor levels in 10 year age groups in 9712 participants without rheumatoid arthritis in the Copenhagen City Heart Study

\begin{tabular}{|c|c|c|c|c|c|}
\hline Autoimmune rheumatic disease & $\begin{array}{l}\text { No of } \\
\text { articipants }\end{array}$ & $\begin{array}{l}\text { No of } \\
\text { events }\end{array}$ & \multicolumn{2}{|c|}{$\begin{array}{c}\text { Hazard ratio } \\
(95 \% \mathrm{CI})\end{array}$} & $\begin{array}{l}\text { Hazard ratio } \\
(95 \% \mathrm{Cl})\end{array}$ \\
\hline Rheumatoid arthritis & 9712 & 183 & & $\rightarrow$ & 3.3 (2.7 to 4.0 ) $P<0.0001$ \\
\hline Sjögren's syndrome & 9710 & 25 & & & $1.8(0.8$ to 4.1$) \mathrm{P}=0.17$ \\
\hline Systemic lupus erythematosus & 9710 & 16 & & & $2.5(0.9$ to 6.6$) P=0.06$ \\
\hline Systemic sclerosis & 9712 & 7 & & & $3.1(1.0$ to 9.3$) \mathrm{P}=0.05$ \\
\hline Polymyositis or dermatomyositis & 9712 & 0 & & & No events \\
\hline & & 0.25 & 0.5 & 1 & 5 \\
\hline
\end{tabular}

Fig 2 Risk of autoimmune rheumatic diseases as a function of doubling of rheumatoid factor level in 9712 participants in the Copenhagen City Heart Study followed for up to 28 years. All hazard ratios were multivariable adjusted (see text for details)

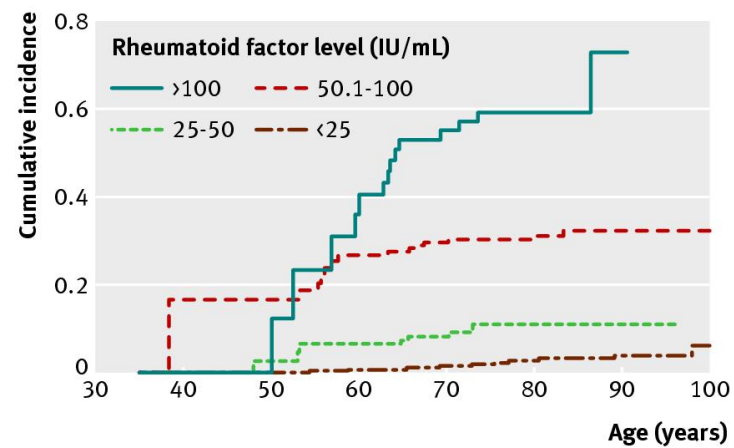

Fig 3 Kaplan-Meier cumulative incidence of rheumatoid arthritis for four categories of baseline level of rheumatoid factor as a function of age in 9712 participants in the Copenhagen City Heart Study followed for up to 28 years 
Any rhe umatoid arthritis hospitalisation 28 years of follow-up

Rheumatoid factor $(\mathrm{IU} / \mathrm{mL})$ No of
participants

No of

25-50

50.1-100

$>100$

9294

176

187

55

147
8
14
14

10 years of follow-up

Rheumatoid

factor (IU/mL)

$<25$

25-50

50.1-100

$>100$

No of
participant
9294
176
187
55

Nov
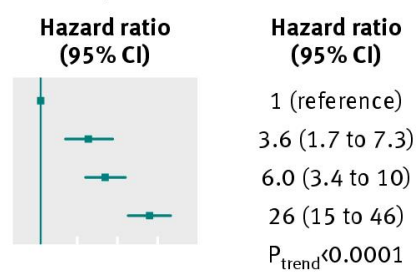

$P_{\text {trend }}<0.0001$

\section{Hazard ratio} $(95 \% \mathrm{Cl})$

41

4

8

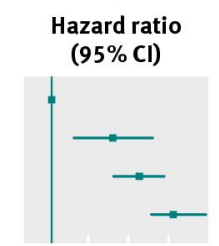

0.513103590
1 (reference)

6.0 (2.1 to 17$)$

14 (6.7 to 28 )

39 (18 to 85$)$

$P_{\text {trend }}<0.0001$

$\geq 2$ rheumatoid arthritis hospitalisations

\section{8 years of follow-up}

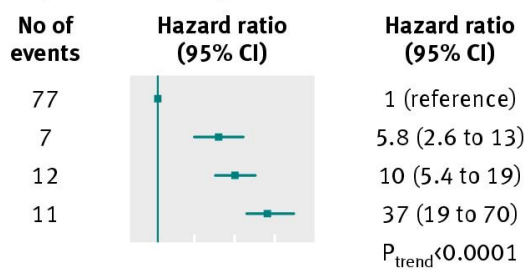

\section{0 years of follow-up}

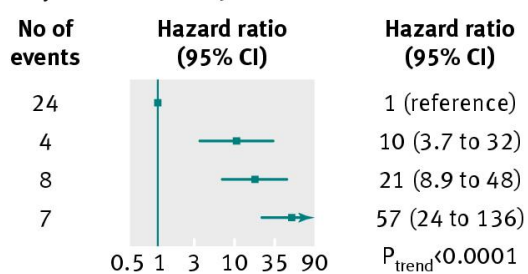

Fig 4 Risk of rheumatoid arthritis as a function of rheumatoid factor level in 9712 participants in the Copenhagen City Heart Study by length of follow-up (full 28 years or first 10 years) and number of hospitalisations for rheumatoid arthritis. All hazard ratios were multivariable adjusted (see text for details)
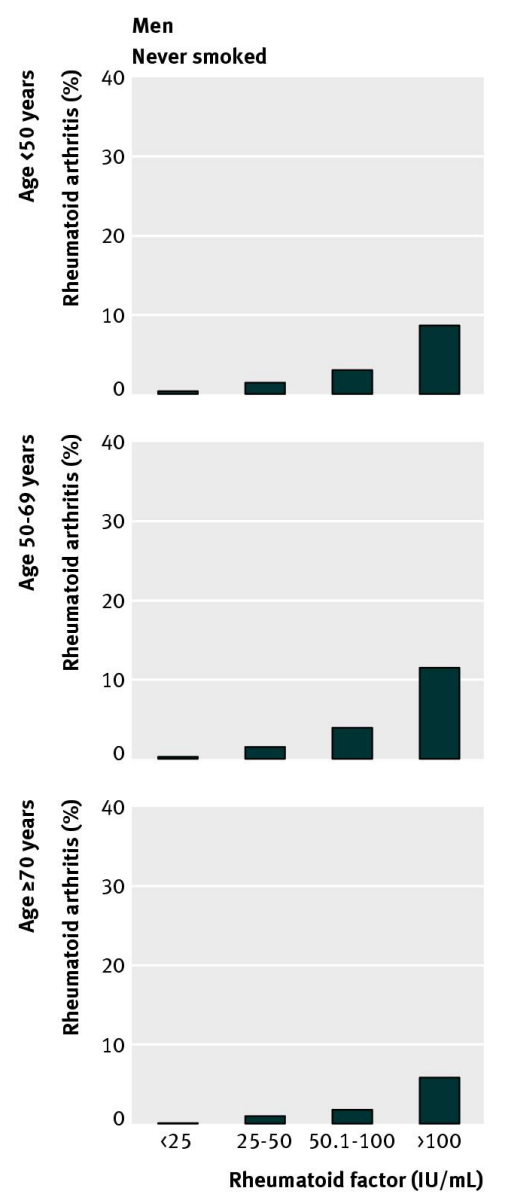

Ever smoked

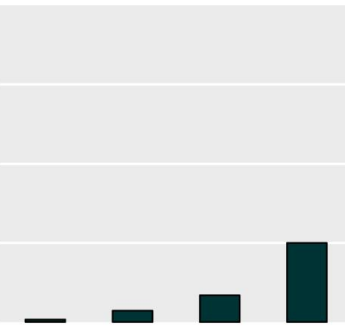

Women

Never smoked
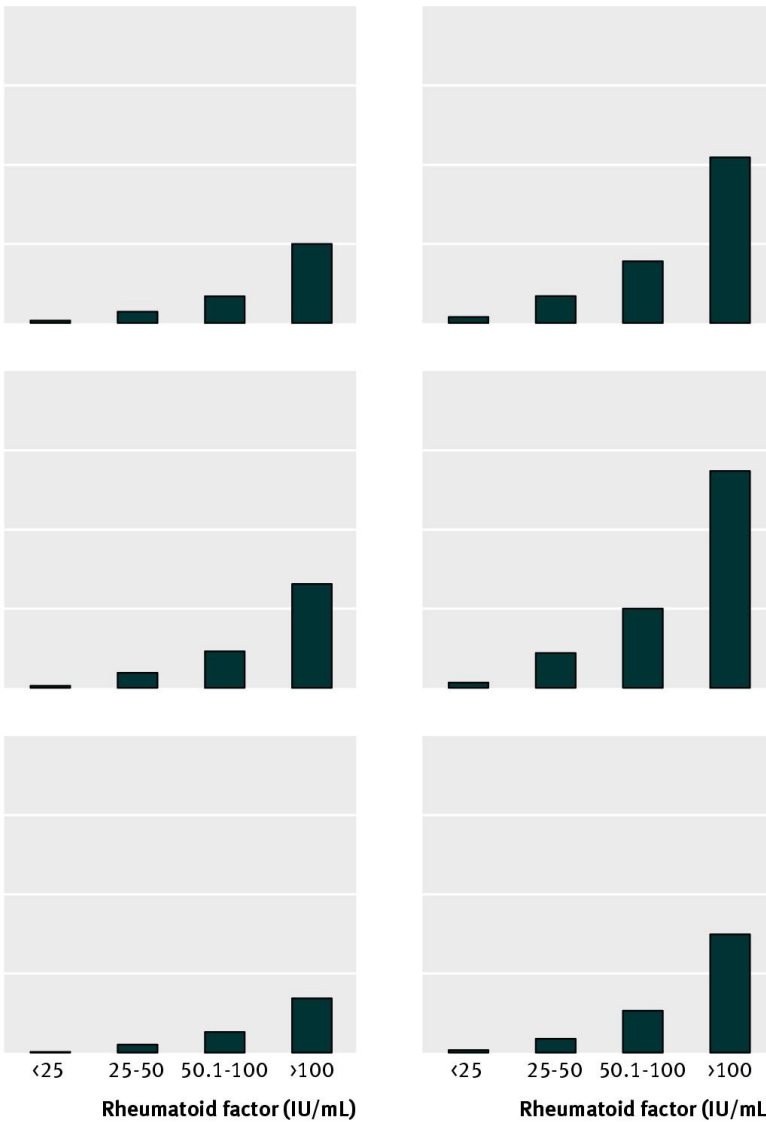
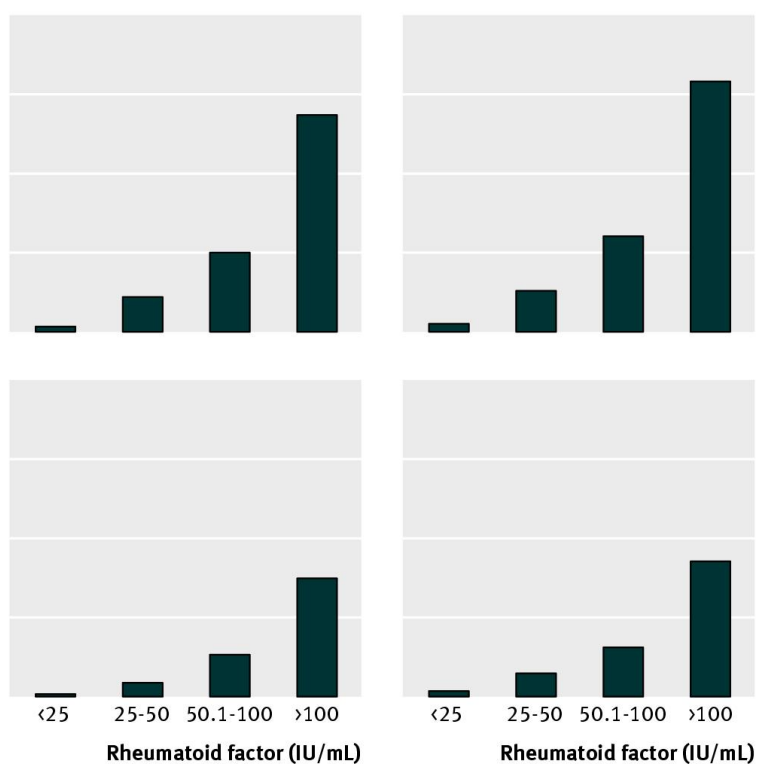

Fig 5 Absolute 10 year risk of rheumatoid arthritis in 9712 participants in the Copenhagen City Heart Study as a function of rheumatoid factor level, age, sex, and smoking status 